Check for updates

Cite this: RSC Adv., 2019, 9, 18087

\title{
Synthesis of hierarchical ZSM-12 nanolayers for levulinic acid esterification with ethanol to ethyl levulinate $\uparrow$
}

\author{
Pannida Dugkhuntod, Thidarat Imyen, (D) * Wannaruedee Wannapakdee, \\ Thittaya Yutthalekha, Saros Salakhum and Chularat Wattanakit (D)
}

\begin{abstract}
Hierarchical ZSM-12 nanolayers have been successfully synthesized via a one-pot hydrothermal process using dimethyloctadecyl[3-(trimethoxysilyl)propyl]ammonium chloride (TPOAC) as a secondary organic structure-directing agent (OSDA). The as-synthesized ZSM-12 samples were characterized by means of XRD, SEM, TEM, $\mathrm{N}_{2}$ physisorption, and $\mathrm{NH}_{3}-\mathrm{TPD}$. This clearly demonstrates that the TPOAC content and the crystallization time are crucial parameters for the formation of nanolayered structures. The presence of such a structure significantly improves the mesoporosity of ZSM-12 by generating interstitial mesopores between nanolayers, eventually resulting in enhancing external surface areas and mesopore volumes, and subsequently promoting the molecular diffusion inside a zeolite framework. To illustrate its advantages as a heterogeneous catalyst, hierarchical ZSM-12 nanolayers were applied in the catalytic application of an esterification of levulinic acid with ethanol to ethyl levulinate. Interestingly, hierarchical ZSM-12 nanolayers exhibit an improvement of catalytic activity in terms of levulinic acid conversion (78.5\%) and ethyl levulinate selectivity (98.7\%) compared with other frameworks of hierarchical zeolite nanosheets, such as ZSM-5 and FAU. The example reported herein demonstrates an efficient way to synthesize a unidimensional pore zeolite with hierarchical nanolayered structure via a dual template method and also opens up perspectives for the application of different hierarchical porous systems of zeolites in the bulky-molecule reactions such as in the case of levulinic acid esterification with ethanol.
\end{abstract}

\author{
Received 29th April 2019 \\ Accepted 3rd June 2019 \\ DOI: $10.1039 / c 9 r a 03213 d$ \\ rsc.li/rsc-advances
}

\section{Introduction}

Ethyl levulinate (EL), a short-chain fatty ester, is an important compound in the flavouring and fragrance industries. In addition, this compound is attractive as a diesel miscible biofuel (DMB), as it can be used up to $5 \mathrm{wt} \%$ for regular diesel car engines. ${ }^{1}$ Typically, EL can be produced by the esterification of levulinic acid (LA) with bioethanol, which is an attractive green process, as LA and bioethanol are easily obtained from biomass. ${ }^{2}$ Typically, the esterification of LA with ethanol can proceed using both soluble and solid acid catalysts. For the esterification reaction in liquid-phase, mineral acids such as $\mathrm{H}_{2} \mathrm{SO}_{4},{ }^{3} \mathrm{HCl},{ }^{4}$ and $\mathrm{H}_{3} \mathrm{PO}_{4}$ (ref. 5) have been used as homogeneous acid catalysts. However, these catalysts are corrosive but are cost-effective. On the other hand, solid catalysts are more

Department of Chemical and Biomolecular Engineering, School of Energy Science and Engineering, Nanocatalysts and Nanomaterials for Sustainable Energy and Environment Research Network of NANOTEC, Vidyasirimedhi Institute of Science and Technology, Rayong 21210, Thailand.E-mail: thidarat.i@vistec.ac.th

$\dagger$ Electronic supplementary information (ESI) available: $\mathrm{NH}_{3}$-TPD profiles and their deconvolution (Fig. S1), ${ }^{29} \mathrm{Si}$-NMR (Fig. S2), BJH pore size distribution (PSD) (Fig. S3), acetic acid conversion and ethyl acetate selectivity (Table S1), $\mathrm{NH}_{3}$-TPD data (Table S2) and textural properties (Table S3). See DOI: 10.1039/c9ra03213d desirable, as they provide many advantages, such as ease of separation from reaction products, the efficient reusability of the catalysts, and less corrosion of reactor materials. Therefore, various solid catalysts have been extensively developed over the past decade as potential catalysts for this reaction, such as zeolites, ${ }^{2}$ sulfated oxides $\left(\mathrm{ZrO}_{2}, \mathrm{SnO}_{2}, \mathrm{TiO}_{2}\right){ }^{6-8}$ Amberlyst-15, ${ }^{9}$ SAC-13 ( $\mathrm{SiO}_{2}$-supported Nafion $){ }^{\mathbf{1 0}}$ heteropoly acids, ${ }^{\mathbf{1 1}}$ and sulfonated carbons. ${ }^{12}$

Among these solid catalysts, zeolites are crystalline aluminosilicate materials, having the acidic properties and a welldefined three-dimensional crystalline structure with uniformly sized nano-channels. Owing to their outstanding properties, such as high surface area, shape selectivity, high thermal/ chemical stability, as well as tunable acid properties, they are indispensable materials for various applications ranging from separation, adsorption to catalysis. ${ }^{\mathbf{1 3}, \mathbf{1 4}}$ Contrary to non-zeolitic catalysts, the catalytic performance of zeolites for esterification of LA with ethanol not only depends on the acidity of catalysts but also relates to the porous structure of zeolites. ${ }^{2}$ Hence, it is essential to use zeolites that have larger cavities to improve the catalytic performance in the esterification of LA with ethanol, involving a bulky molecule as a product. Therefore, it is reasonable to conclude that the structure-property 
relationship of a catalyst is a key factor for further improvement in productivity and selectivity. ${ }^{2}$

ZSM-12 (MTW) is a silica rich zeolite, composing of onedimensional 12-membered ring channel system with unique pore openings of $5.7 \AA \times 6.1 \AA .{ }^{15}$ Since its pore size is slightly higher than that of ZSM-5, it can be used for the shape selective conversion of large molecules, which cannot easily penetrate into MFI framework. In addition, due to its acidic properties, ZSM-12 has been suggested as a useful catalyst in many reactions such as isomerization, ${ }^{16}$ hydroisomerization, ${ }^{17}$ and hydrocracking. ${ }^{18}$ Nevertheless, there is no report according to using this zeolite as the catalyst for EL production so far. Therefore, with its larger pore dimension compared with other zeolites and suitable acidity, it would benefit from using this material as a novel and useful catalyst in esterification of LA with ethanol.

Unfortunately, it is well-known that a microporous structure of zeolites restricts the diffusion of guest molecules within their porous network, leading to the low accessibility of active sites, mass transport limitations, and catalyst deactivation. ${ }^{14}$ In order to ease these problems, zeolites exhibiting hierarchical porous structures have been developed. These materials exhibit the presence of at least one additional porous system, such as mesoporous and macroporous systems. ${ }^{19}$ These materials may benefit to improve the diffusion in the typical microporous network because the created mesoporosity can facilitate the mass transport and lead to a more efficient use for catalytic applications. ${ }^{20}$ The hierarchical zeolites can be obtained by several approaches, such as desilication, ${ }^{19,21}$ dealumination, ${ }^{21}$ steaming, ${ }^{22}$ recrystallization, ${ }^{23,24}$ and templating approach..$^{25,26}$ Among them, the use of a template has been considered as one of the most the effective approaches to synthesize hierarchical zeolites.

Recently, the hierarchical zeolite nanosheets have been successfully prepared and attracted much attention. Since these materials are composed of sheet-like crystals with the thickness in the nanometer range, they provide large surface areas as well as high pore volumes, which are derived from the presence of interstitial mesopores between adjacent nanocrystals. ${ }^{27}$ One of the most efficient approaches for the preparation of hierarchical zeolite nanosheets is based on the use of an organic structure-directing agent (OSDA) to control microporous structures of zeolites and nanocrystals formation..$^{28-32}$

Choi et $a l .{ }^{28}$ utilized a diquaternary ammonia-type surfactant $\left(\mathrm{C}_{22} \mathrm{H}_{45}-\mathrm{N}^{+}\left(\mathrm{CH}_{3}\right)_{2}-\mathrm{C}_{6} \mathrm{H}_{12}-\mathrm{N}^{+}\left(\mathrm{CH}_{3}\right)_{2}-\mathrm{C}_{6} \mathrm{H}_{13}\right)$ for the synthesis of MFI nanosheets. The diammonium head group performs as a structure-directing agent for MFI structure, whereas the hydrophobic long-chain tails forming a micellar structure restrict the growth of zeolite and create $2 \mathrm{D}$ zeolite nanolayers. Meanwhile, Na et $a l .{ }^{27}$ proposed the use of different cyclic ammonium compounds as the OSDAs to synthesize BEA, MTW, and MFI zeolite structures with nanocrystalline morphologies. Among various OSDAs utilized for the zeolite preparation, an organosilane surfactant is one of the outstanding candidates for directing the formation of zeolite with layered structure. The strong interaction between the molecules of organosilane and the growing zeolite crystals is a key aspect to prevent a phase separation in a mixture solution of the OSDAs and the zeolite gel. Recently, various types of nanosheet assemblies such as FER and FAU were successfully prepared by utilizing an organosilane with 18-carbon alkyl chains, namely dimethyloctadecyl [3-(trimethoxysilyl)propyl] ammonium chloride or TPOAC as the mesopore template. ${ }^{33-35}$ However, to the best of our knowledge, it has not yet been reported in the use of this type of organosilanes as the co-SDA for the synthesis of unidimensional ZSM12 porous nanolayers.

Therefore, in order to compliment the use of organosilanes for synthesizing other hierarchical zeolites nanolayers besides FAU and FER nanosheets, a simple and practical approach to prepare hierarchical ZSM-12 using TPOAC as the co-SDA is demonstrated herein. Since cyclic ammonium compounds were also found as the effective SDA to generate MTW zeolite structures with nanocrystalline morphologies, it is beneficial to combine both cyclic ammonia compound and TPOAC as the primary and the secondary SDA for the synthesis of hierarchical ZSM-12 nanolayer assemblies, respectively. Moreover, the roles of synthesis parameters including TPOAC content and crystallization time on the product characteristic were investigated. Additionally, the exceptional catalytic efficiency of synthesized hierarchical ZSM-12 nanolayer for bulky-molecules reaction, namely, levulinic acid esterification with ethanol to ethyl levulinate was also demonstrated. To further investigate the effect of one-dimensional 12-membered ring channel system of ZSM-12 on the catalytic performance, different zeolite nanosheets with various framework structures such as ZSM-5 and FAU, composing of different dimensional porous systems were used for the comparison in terms of catalytic activity and selectivity.

\section{Experimental}

\section{Materials}

Sodium silicate $\left(\mathrm{Na}_{2} \mathrm{Si}_{3} \mathrm{O}_{7}: 26.5 \mathrm{wt} \% \mathrm{SiO}_{2}\right.$, and $10.6 \mathrm{wt} \% \mathrm{Na}_{2} \mathrm{O}$, Merck), aluminium sulphate $\left(\mathrm{Al}_{2}\left(\mathrm{SO}_{4}\right)_{3} \cdot 18 \mathrm{H}_{2} \mathrm{O}\right.$, Univar, Ajax Finechem), sulfuric acid $\left(\mathrm{H}_{2} \mathrm{SO}_{4}: 96 \%\right.$, RCI Labscan), sodium hydroxide ( $\mathrm{NaOH}$ : 98\%, Carlo Erba), $\alpha, \alpha^{\prime}$-dichloro- $p$-xylene (98\%，TCI), $N, N, N^{\prime}, N^{\prime}$-tetramethyl-1,6-hexanediamine (98\%, TCI), and dimethyloctadecyl[3-(trimethoxysilyl)propyl]ammonium chloride (TPOAC: $42 \mathrm{wt} \%$ in methanol, Aldrich) were used as starting materials for the synthesis of hierarchical ZSM-12 nanolayers. Levulinic acid $(>97 \%$, TCI), toluene $(\geq 99.9 \%$, Merck), ethanol (99.9\%, QRec), and decane ( $\geq 99.9 \%$, TCI) were used for the catalytic activity testing without any further purification.

\section{Synthesis of hierarchical ZSM-12 nanolayer}

Firstly, cyclic diquaternary ammonium (CDM) was synthesized and utilized as an SDA for the synthesis of ZSM-12 zeolite following a previous procedure ${ }^{27}$ with some modifications. Briefly, $N, N, N^{\prime}, N^{\prime}$-tetramethyl-1,6-hexanediamine was mixed with an equimolar amount of $\alpha, \alpha^{\prime}$-dichloro- $p$-xylene in acetonitrile under reflux at $60^{\circ} \mathrm{C}$ for $3 \mathrm{~h}$. Then, the obtained product was filtered and washed with acetonitrile followed by diethyl ether, and dried in a vacuum oven at $50{ }^{\circ} \mathrm{C}$. 
Subsequently, hierarchical ZSM-12 nanolayers were synthesized by using a one-pot hydrothermal method, in which sodium silicate and aluminium sulphate were used as silica and alumina sources, respectively. Meanwhile, CDM and TPOAC was used as an SDA and co-OSDA to control microporous ZSM12 and nanosheets structures, respectively. The molar composition was $30 \mathrm{Na}_{2} \mathrm{O}: 0.625 \mathrm{Al}_{2} \mathrm{O}_{3}: 100 \mathrm{SiO}_{2}: 10 \mathrm{CDM}: 15 \mathrm{H}_{2} \mathrm{SO}_{4}$ : $6000 \mathrm{H}_{2} \mathrm{O}: x$ TPOAC, where $x$ was $0,3,9,12$, and 15 . Typically, the first solution was prepared by diluting sodium silicate with $\mathrm{NaOH}$ solution, while the second solution was prepared by diluting aluminium sulphate with $\mathrm{H}_{2} \mathrm{SO}_{4}$ solution. The two solutions were mixed under vigorous stirring before adding CDM into the mixed solution. After stirring for $1 \mathrm{~h}$, the resultant mixture was transferred to a Teflon lined stainless steel autoclave for hydrothermal treatment at $170{ }^{\circ} \mathrm{C}$ for $24 \mathrm{~h}$. The obtained product was filtered and washed with deionized water, dried at $100{ }^{\circ} \mathrm{C}$ overnight, and calcined at $550{ }^{\circ} \mathrm{C}$ for $6 \mathrm{~h}$. The solid product was converted to $\mathrm{H}^{+}$form via an ion-exchange with $1 \mathrm{M} \mathrm{NH}_{4} \mathrm{NO}_{3}$ solution at $80{ }^{\circ} \mathrm{C}$ for $2 \mathrm{~h}$. The ion-exchange process was repeated for three time. Then, the derived product was washed with deionized water, dried at $100{ }^{\circ} \mathrm{C}$ overnight, and calcined at $550{ }^{\circ} \mathrm{C}$ for $6 \mathrm{~h}$. All the synthesized ZSM-12 nanosheets samples are denoted as ZSM-12-y, where $y$ refers to the molar ratio of $\mathrm{TPOAC} / \mathrm{SiO}_{2}$ in the starting gel.

\section{Characterizations}

X-ray diffraction (XRD) patterns were collected using a Bruker D8 ADVANCE diffractometer with $\mathrm{Cu} \mathrm{K} \alpha$ radiation $(30 \mathrm{kV}$ and 40 $\mathrm{mA}$ ) in the $2 \theta$ range of $5^{\circ}$ to $60^{\circ}$ with a step size of $0.02^{\circ}$ and scan rate of $1^{\circ} \mathrm{min}^{-1}$. The relative crystallinity of zeolite products was determined by the following equation: \% XRD relative crystallinity of the desired sample $=\left(H_{\mathrm{x}} / H_{\mathrm{r}}\right) \times 100$, where $H_{\mathrm{x}}$ and $H_{\mathrm{r}}$ are the heights of the main diffraction peaks of the desired sample and the reference sample, respectively.

The nanolayered structure of the products was confirmed by scanning electron microscopy (SEM, JEOL, JSM-7610F model). Additionally, the morphology of the samples was also monitored by transmission electron microscopy (TEM, JEOL JEM2010) operated with an acceleration of $200 \mathrm{kV}$. Prior each TEM analysis, the sample was suspended in ethanol, followed by evaporation of ethanol on a copper grid coated with a carbon film.

The $\mathrm{Si} / \mathrm{Al}$ ratio of the products was obtained by X-ray fluorescence analysis (XRF, Bruker model S8 TIGER sequential WDXRF).

The textural properties of the products were determined from nitrogen adsorption-desorption isotherm measurement at $-196{ }^{\circ} \mathrm{C}$ by using a MicrotracBEL, BELSORP-max model. The specific surface area $\left(S_{\mathrm{BET}}\right)$ was estimated by the BrunauerEmmett-Teller (BET) method. Meanwhile, the micropore surface area $\left(S_{\text {micro }}\right)$, the external surface area $\left(S_{\text {ext }}\right)$, and the microporous volume $\left(V_{\text {micro }}\right)$ were determined by the $t$-plot method. The total pore volume $\left(V_{\text {total }}\right)$ was obtained from data at $P / P_{0}$ of 0.99 . The pore size distribution was derived from the desorption branch of the Barrett-Joyner-Halenda (BJH) method.
Temperature-programmed desorption of ammonia $\left(\mathrm{NH}_{3}-\right.$ TPD) was used to investigate the acid properties of the obtained products. The measurement was conducted using a BELCAT II instrument equipped with thermal conductivity detectors (TCD). Prior to the measurement, the sample was pre-treated under He flow at $500{ }^{\circ} \mathrm{C}$ for $1 \mathrm{~h}$. After that, the sample was saturated with $\mathrm{NH}_{3}$ at $100{ }^{\circ} \mathrm{C}$ for $30 \mathrm{~min}$. Subsequently, the $\mathrm{NH}_{3}$ desorption was performed from 100 to $550^{\circ} \mathrm{C}$ with the heating rate of $10{ }^{\circ} \mathrm{C} \mathrm{min}^{-1}$.

The coordination environment of $\mathrm{Si}$ atom was investigated using ${ }^{29} \mathrm{Si}$ MAS NMR spectroscopy performed on an AVANCE III HD (400 MHz) Digital NMR spectrometer (Bruker) operating at the magnetic field of $9.4 \mathrm{~T}$.

\section{Catalytic activity testing via an esterification of LA with ethanol}

Esterification of LA with ethanol to EL was carried out using $0.3 \mathrm{~g}$ of the catalyst in a Parr reactor for $24 \mathrm{~h}$. The catalytic activity of the catalysts was tested with an equimolar mixture of LA and ethanol in toluene solvent under stirring (500 rpm) at $100{ }^{\circ} \mathrm{C}$ and autogenous pressure. The reaction mixture was sampled and analyzed by gas chromatograph (GC, Agilent, 7890B) equipped with a FID detector and an Elite-Wax column $(30 \mathrm{~m} \times 0.25 \mathrm{~mm})$ at a certain time of the reaction. For the product analysis, decane was used as an internal standard. Subsequently, LA conversion and EL selectivity were calculated by the following equations:

$$
\begin{aligned}
& \text { LA conversion }(\%)=\frac{\text { mole }^{L} A_{\text {initial }}-\text { mole } \mathrm{LA}_{\text {final }}}{\text { mole }_{\text {LA }} \text { initial }} \times 100 \\
& \text { EL selectivity }(\%)=\frac{\text { mole EL formed }}{\text { mole all products formed }} \times 100
\end{aligned}
$$

\section{Results and discussions}

\section{Synthesis of hierarchical ZSM-12 zeolite nanosheets}

Effects of the TPOAC content in the synthesis gel. X-ray diffraction patterns of ZSM-12 nanolayers synthesized with different TPOAC/ $\mathrm{SiO}_{2}$ molar ratios are presented in Fig. 1 . The samples obtained with $\mathrm{TPOAC} / \mathrm{SiO}_{2}$ ratio ranging from 0 to 0.12 exhibit the characteristic diffraction peaks of MTW $\left(7.2^{\circ}, 8.8^{\circ}\right.$, $20.7^{\circ}$, and $\left.23.1^{\circ}\right)^{15,36}$ without the interference of other crystalline phases, confirming that ZSM-12 zeolite was successfully synthesized. However, the intensities of these diffraction peaks decrease when TPOAC/ $\mathrm{SiO}_{2}$ ratio was increased, indicating that the crystallinity of ZSM-12 decreases as a function of the TPOAC content, in agreement with what have been reported in previous works. ${ }^{33,34,36}$ The reason for a decrease in crystallinity can be related to the reduced microporous feature, which is the main characteristic of zeolite. Typically, the relative crystallinity is determined based on the summation of the heights of the main diffraction peaks of ZSM-12 $\left(7.2^{\circ}, 8.8^{\circ}, 20.7^{\circ}\right.$, and $\left.23.1^{\circ}\right)$ of the desired sample with respect to those of the reference sample. ${ }^{36,37}$ In this case, the ZSM-12 sample synthesized in the 


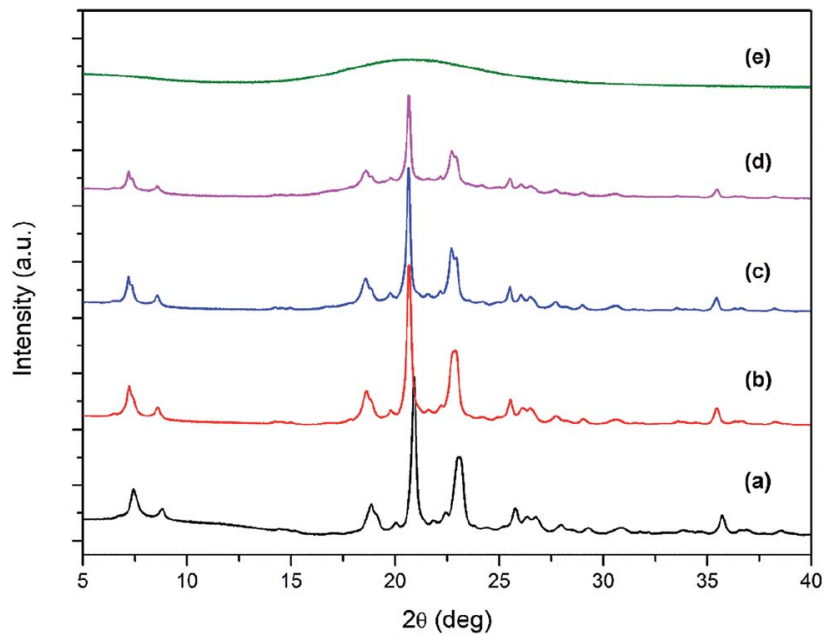

Fig. 1 XRD patterns of ZSM-12 zeolites synthesized with different $\mathrm{TPOAC} / \mathrm{SiO}_{2}$ molar ratios: (a) ZSM-12-0, (b) ZSM-12-0.03, (c) ZSM-120.09 , (d) ZSM-12-0.12, and (e) ZSM-12-0.15

absence of TPOAC as a secondary SDA (ZSM-12-0) was chosen as the reference sample. For instance, the relative crystallinity of ZSM-12 products decreases from 100 to 95.02, 85.07, and $66.32 \%$ for ZSM-12-0.03, ZSM-12-0.09, and ZSM-12-0.12, respectively. Obviously, at the TPOAC/ $\mathrm{SiO}_{2}$ molar ratio of 0.15 , there is no any characteristic peaks of MTW and only amorphous phase is observed. It is therefore reasonable to concern that the optimum amount of TPOAC is a crucial parameter to control the nanolayered configuration.

The morphology of the synthesized ZSM-12 was revealed by SEM images as illustrated in Fig. 2. For the sample obtained without TPOAC (ZSM-12-0), the agglomeration of nanosized crystals without the formation of nanolayers of ZSM-12 was detected and similar to what have been described in the previous literature $^{27}$ (Fig. 2(a)). In strong contrast to this, with the addition of TPOAC as the co-SDA with the suitable amount, the assemblies of ZSM-12 nanolayers with a crystalline morphology were observed, indicating that the presence of TPOAC is crucial for the formation of ZSM-12 nanolayered structures.

However, the formation of such structures strongly depends on the content of TPOAC in the synthesis gel. For example, as the TPOAC/ $/ \mathrm{SiO}_{2}$ ratio is changed from 0 to 0.03 , the nanosized crystals become the brick-like crystals (Fig. 2(b)). Moreover, upon increasing the $\mathrm{TPOAC} / \mathrm{SiO}_{2}$ ratio to 0.09 , the average particle size is increased and the well-defined nanolayerassembled ZSM-12 having the thickness of each nanolayer around $20-25 \mathrm{~nm}$ is obtained (Fig. 2(c)), indicating that the intergrowth of nanolayers is predominant to form nanolayer assembly. ${ }^{33,34}$ Nevertheless, with further increase of the TPOAC/ $\mathrm{SiO}_{2}$ ratio to 0.12 , nanolayer assemblies are revealed in the simultaneous presence of the amorphous phases and crystalline phase of ZSM-12 (Fig. 2(d)). The presence of the amorphous phase could be indicated by a significantly lower crystallinity and broad hump at $2 \theta$ around 20 (see Fig. 1). Finally, when the $\mathrm{TPOAC} / \mathrm{SiO}_{2}$ molar ratio reaches 0.15 , the nanolayered
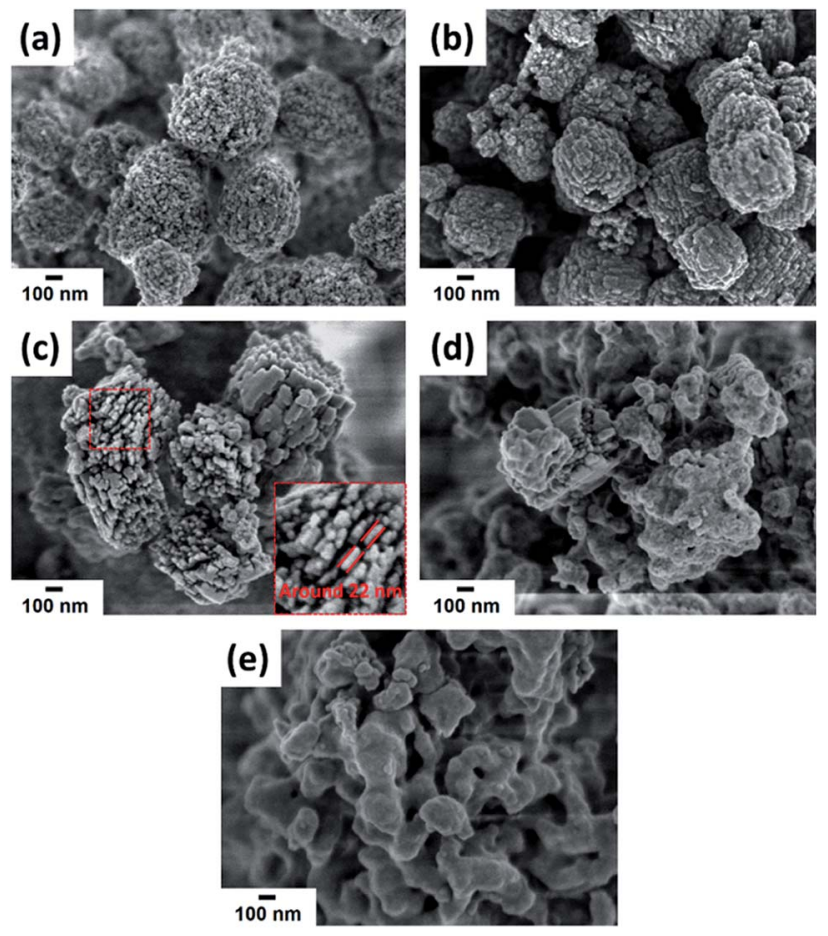

Fig. 2 SEM images of ZSM-12 zeolites synthesized with different TPOAC/SiO 2 molar ratios: (a) ZSM-12-0, (b) ZSM-12-0.03, (c) ZSM-120.09, (d) ZSM-12-0.12, and (e) ZSM-12-0.15.

morphology is completely destroyed (Fig. 2(e)), and the absence of diffraction peaks in XRD patterns can allow us to assume that the excess amount of TPOAC can generate an amorphous phase instead of a crystalline zeolite (see Fig. 1). These observations suggest that too high TPOAC content can inhibit crystal growth and also disrupt the self-assembly of nanosheets.

The excessive amount of TPOAC can cause the adsorptive competition of the organosilane surfactant on zeolite precursor surfaces, eventually resulting in the loss of the long-range order of zeolite structures. ${ }^{38}$ It is therefore reasonable to propose the formation process of ZSM-12 nanolayer assemblies as

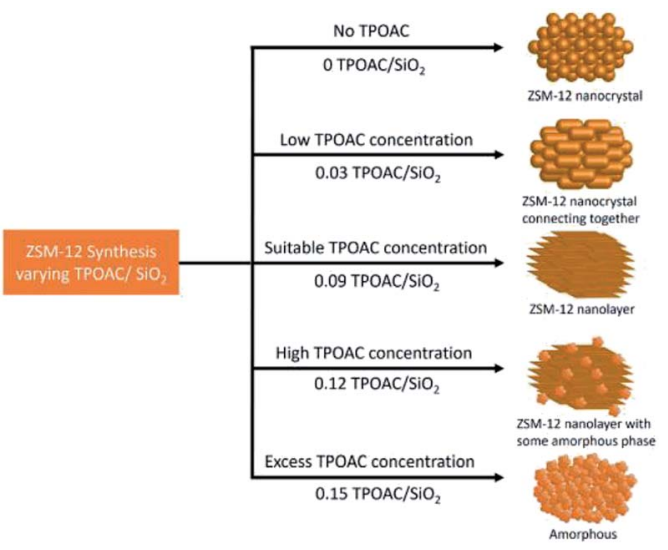

Scheme 1 The formation of ZSM-12 nanolayer assemblies as a function of TPOAC concentration. 
demonstrated in Scheme 1. Since, the introduction of TPOAC has a considerable influence on the crystallization of ZSM-12 nanolayers, the suitable amount of TPOAC is required in order to obtain homogeneous ZSM-12 nanolayers with the welldefined structure as well as high crystallinity. Therefore, as above-mentioned results, the most suitable $\mathrm{TPOAC} / \mathrm{SiO}_{2}$ molar ratio under the present synthesis circumstance is 0.09 .

To further study the effects of TPOAC in the synthesis gel on the textural properties of the crystalline ZSM-12 products, $\mathrm{N}_{2}$ adsorption-desorption experiments were carried out on the crystalline ZSM-12 samples obtained with $\mathrm{TPOAC} / \mathrm{SiO}_{2}$ molar ratios of 0 to 0.09 . The textural properties of the samples are summarized in Table 1. As depicted in Fig. 3, both ZSM-120 and ZSM-12-0.09 samples exhibit type-IV $\mathrm{N}_{2}$ isotherms with a hysteresis loop, ${ }^{39}$ confirming the simultaneous presence of micropores and meso/macropores in the obtained ZSM-12 products. The steep $\mathrm{N}_{2}$ uptakes at low $P / P_{0}$ relates to the microporous structure, while the hysteresis loop existing in the region $0.45<P / P_{0}<0.95$ corresponds to capillary condensation in mesopores. ${ }^{27,40}$ Interestingly, ZSM-12-0.03 sample exhibits type-I $\mathrm{N}_{2}$ isotherm, a typical of microporous materials, with a thin hysteresis loop between 0.4 and 0.8 , indicating to the presence of some mesoporosity as shown in Table $1 .{ }^{36}$

Unsurprisingly, the mesoporosity of the sample synthesized in the absence of TPOAC (ZSM-12-0) is created by the use of a single SDA of cyclic diquaternary ammonium (CDM) to control the MTW framework. The high affinity of the organic cyclic diammonia with silicate species and the intergrowths lead to the agglomeration of nanocrystalline ZSM-12, yielding mesopores between adjacent nanoparticles. ${ }^{27}$ The introduction of TPOAC as the secondary template directly affects the fraction of mesopores in ZSM-12 products. Typically, the presence of TPOAC can inhibit the crystal growth at specific direction, resulting in the generation of $2 \mathrm{D}$ zeolite nanolayers and interstitial mesopores between these nanolayers. However, as discussed above, the content of TPOAC should be suitable in order to obtain well-defined and homogeneous ZSM-12 nanolayers with high crystallinity. For instance, when the TPOAC/SiO molar ratio is changed from 0 to 0.03 , the nanosized crystals become the brick-like crystals (Fig. 2(a) and (b)). Subsequently, with a bigger size of brick-like crystals (Fig. 2(b) and Scheme 1), the interparticle voids become smaller and thus the fraction of mesopores is lower. Hence, the microporous feature is predominant, while only small fraction of mesopores is present in the ZSM-12-0.03 sample, as evidenced by $\mathrm{N}_{2}$ isotherm and textural properties shown in Fig. 3 and Table 1, respectively.

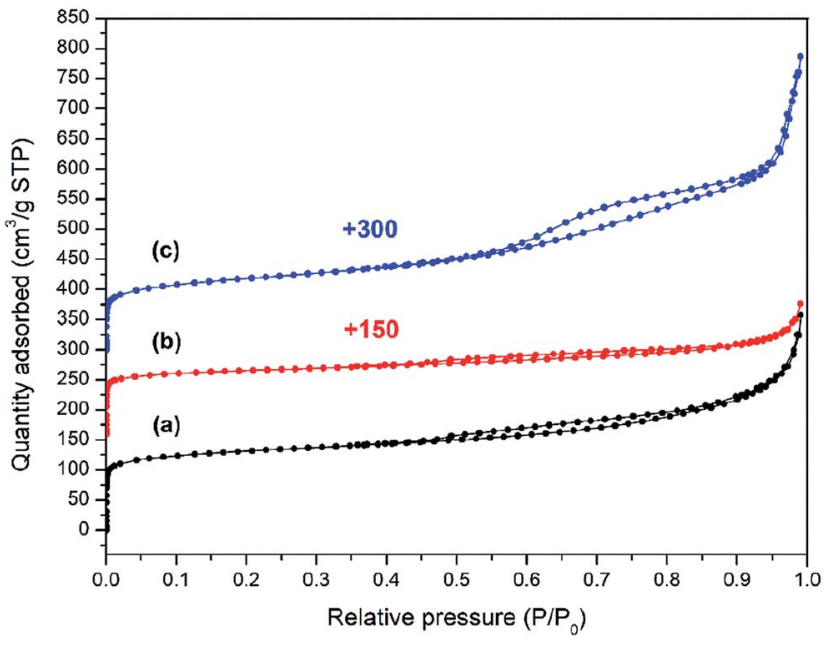

Fig. $3 \mathrm{~N}_{2}$ adsorption-desorption isotherms of ZSM-12 zeolites synthesized with different $\mathrm{TPOAC} / \mathrm{SiO}_{2}$ molar ratios: (a) ZSM-12-0, (b) ZSM-12-0.03, and (c) ZSM-12-0.09.

On the other hand, with the addition of TPOAC with the suitable amount (i.e. TPOAC/SiO ${ }_{2}$ molar ratio of 0.09 ), the fraction of mesopores in the ZSM-12 product becomes higher, as demonstrated by a manifest hysteresis loop presenting in the $\mathrm{N}_{2}$ isotherms of ZSM-12-0.09 sample (Fig. 3). As expected, the external surface area of ZSM-12 products increases with an increasing of TPOAC/SiO 2 ratio to 0.9 , for example; the external surface area increases to $278 \mathrm{~m}^{2} \mathrm{~g}^{-1}$ for ZSM-12-0.09. In addition, the mesopore volume and the total pore volume of ZSM-120.09 increase to 0.71 and $0.75 \mathrm{~cm}^{3} \mathrm{~g}^{-1}$, respectively, compared with ZSM-12 products obtained with lower TPOAC content. As the TPOAC/ $/ \mathrm{SiO}_{2}$ ratio is increased to 0.09 , the crystal thickness becomes smaller and nanolayer-assembled ZSM-12 is subsequently obtained (Fig. 2(c)). The above results confirm that the introduction of TPOAC as the secondary template can improve the mesoporosity of ZSM-12 zeolite. This behaviour relates to the fact that the nanosized crystals become sheet-like and the assembly of nanosized sheet-like crystals, which can enhance the interstitial mesoporosity.

The acid properties of synthesized ZSM-12 were determined by $\mathrm{NH}_{3}$-TPD measurement as shown in Fig. 4 . $\mathrm{NH}_{3}$-TPD profiles of all samples were decomposed into three peaks at around 180-200 ${ }^{\circ} \mathrm{C}, 250-310{ }^{\circ} \mathrm{C}$, and $360-400{ }^{\circ} \mathrm{C}$ (Fig. S1 $\dagger$ ), corresponding to weak, medium, and strong acid sites, respectively. ${ }^{36,40}$ The desorption temperatures and the corresponding

Table 1 Textural properties and the Si/Al ratio of the ZSM-12 products

\begin{tabular}{|c|c|c|c|c|c|c|c|c|}
\hline Sample & $S_{\mathrm{BET}}{ }^{a}$ & $S_{\text {micro }}^{b}$ & $S_{\text {ext }}^{c}$ & $V_{\text {total }}{ }^{d}$ & $V_{\text {micro }}^{e}$ & $V_{\text {ext }}^{f}$ & $V_{\text {ext }} / V_{\text {total }}{ }^{g}$ & $\mathrm{Si} / \mathrm{Al}^{h}$ \\
\hline ZSM-12-0 & 484 & 307 & 176 & 0.54 & 0.11 & 0.43 & 0.80 & 52.5 \\
\hline ZSM-12-0.03 & 393 & 278 & 115 & 0.33 & 0.10 & 0.23 & 0.70 & 58.7 \\
\hline
\end{tabular}

${ }^{a} S_{\mathrm{BET}}$ : BET specific surface area. ${ }^{b} S_{\text {micro }}$ : microporous surface area. ${ }^{c} S_{\text {ext }}$ : external surface area. ${ }^{d} V_{\text {total }}:$ total pore volume. ${ }^{e} V_{\text {micro }}:$ micropore volume. ${ }^{f} V_{\text {ext }}=V_{\text {total }}-V_{\text {micro }}$; all surface areas and pore volumes are in the units of $\mathrm{m}^{2} \mathrm{~g}^{-1}$ and $\mathrm{cm}^{3} \mathrm{~g}^{-1}$, respectively. ${ }^{g}$ Fraction of mesopore volume. ${ }^{h} \mathrm{Si} / \mathrm{Al}$ ratio obtained by XRF. 
acid amounts of all samples are summarized in Table 2 . Compared with ZSM-12-0 as the reference sample, the acid strength of ZSM-12 nanolayer assemblies is slightly weaker, as indicated by a peak shifting to lower desorption temperatures when TPOAC/SiO ${ }_{2}$ molar ratio is increased from 0 to 0.03 and 0.09 , respectively (Table 2 ). In addition, the total number of acid sites represented by the area of $\mathrm{NH}_{3}$-TPD peaks of ZSM-12 products is significantly decreased when TPOAC content is increased from 0 to 0.03 and 0.09 , respectively. A lower acidity of ZSM-12 nanolayer assemblies compared with that of the reference ZSM-12 is correlated with a higher $\mathrm{Si} / \mathrm{Al}$ ratio caused by the presence of TPOAC in the synthesis gel. Since TPOAC can also act as a part of silica source, ${ }^{\mathbf{4 0}}$ the addition of TPOAC tends to introduce more silicon atoms in the zeolite framework, resulting in increasing the $\mathrm{Si} / \mathrm{Al}$ ratio and thus decreasing the number of acidic sites. For instance, a Si/Al ratio increases from 52.5 to 69.7 when TPOAC/ $\mathrm{SiO}_{2}$ ratio increases from 0 to 0.09 (see Table 1).

Effects of the crystallization time. The formation of ZSM-12 nanolayer assembly was investigated by varying the crystallization time from 2 to $24 \mathrm{~h}$, while $\mathrm{TPOAC} / \mathrm{SiO}_{2}$ ratio was fixed at 0.09 , as it is the most suitable ratio to obtain the well-defined nanolayered structure with high crystallinity. A change of morphology as a function of crystallization time was observed by SEM and TEM images as illustrated in Fig. 5 and 6, respectively. At the crystallization period of 2 to $6 \mathrm{~h}$, the aluminosilicate species are initially formed as the amorphous gel with

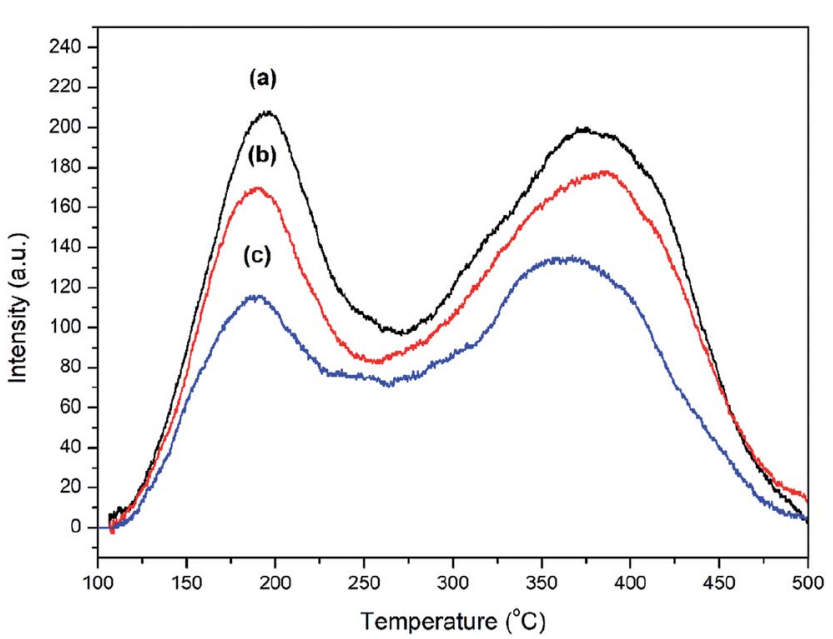

Fig. $4 \mathrm{NH}_{3}$-TPD profiles of ZSM-12 zeolites synthesized with different $\mathrm{TPOAC} / \mathrm{SiO}_{2}$ molar ratios: (a) ZSM-12-0, (b) ZSM-12-0.03, and (c) ZSM-12-0.09. a spongy-like morphology (Fig. 5(a)-(c)). The amorphous material obtained in this crystallization period was also confirmed by XRD patterns after $6 \mathrm{~h}$ of crystallization (Fig. 7). During the crystallization time of $12 \mathrm{~h}$, the nanolayers start to form under the directing of TPOAC and gradually assemble to nanosheet assemblies as demonstrated by SEM and TEM images (Fig. 5(d) and Fig. 6(d)). However, there is still amorphous phase detected in this period (Fig. 5(d)). It is well known that the zeolite crystallization occurs after the dissolution of amorphous phase. ${ }^{27}$

TEM images as illustrated in Fig. 6 also provide the information regarding the formation of ZSM-12 nanolayers as a function of crystallization time. For instance, at the initial stage of the crystallization ( 0 to $6 \mathrm{~h}$ ), the sample is present as an amorphous aluminosilicate gel (Fig. 6(a)-(c)). As the crystallization time is increased to $12 \mathrm{~h}$, a crystalline phase starts to appear (Fig. 6(d)). Subsequently, after the crystallization of $24 \mathrm{~h}$, the amorphous phase is completely transformed to the multilayer splice of ZSM-12 nanosheets, which can also be confirmed by high crystallinity $(85.07 \%)$.
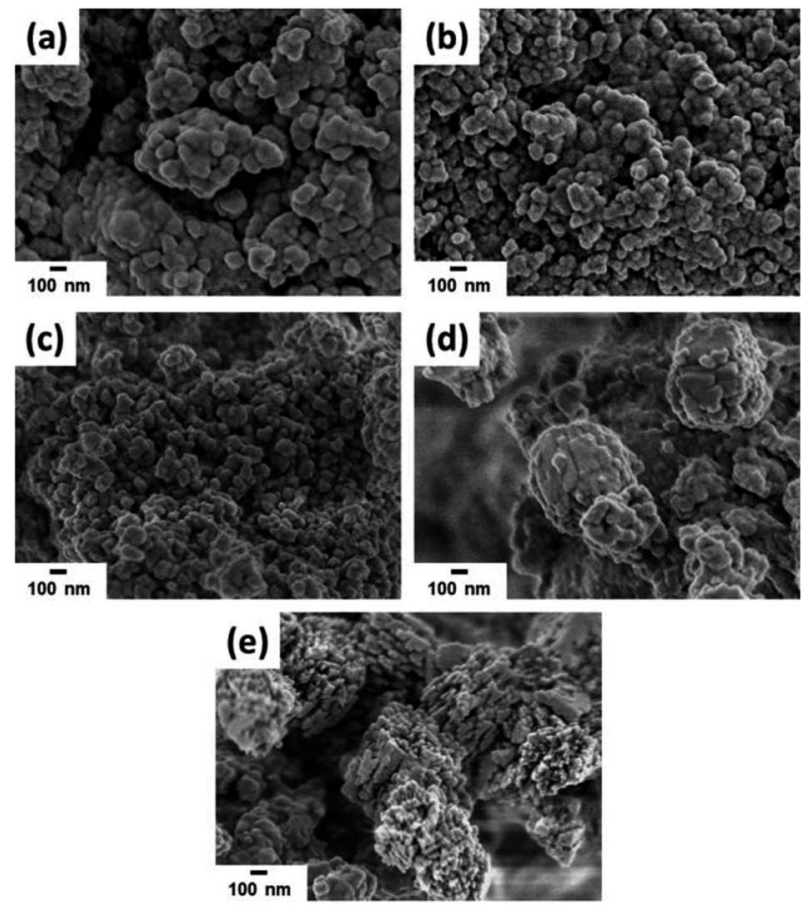

Fig. 5 SEM images of ZSM-12-0.09 samples obtained at different crystallization time: (a) $2 \mathrm{~h}$, (b) $4 \mathrm{~h}$, (c) $6 \mathrm{~h}$, (d) $12 \mathrm{~h}$, and (e) $24 \mathrm{~h}$.

Table $2 \mathrm{NH}_{3}$-TPD data and the acid amount of synthesized ZSM-12 zeolites

\begin{tabular}{|c|c|c|c|c|c|c|c|}
\hline \multirow[b]{2}{*}{ Sample } & \multicolumn{3}{|l|}{$T_{\text {peak }}\left({ }^{\circ} \mathrm{C}\right)$} & \multicolumn{4}{|c|}{ Acid amount $\left(\mathrm{mmol} \mathrm{g}^{-1}\right)$} \\
\hline & LT peak & MT peak & HT peak & Weak acidity & Medium acidity & Strong acidity & Total acidity \\
\hline ZSM-12-0.03 & 185.5 & 285.4 & 387.7 & 0.058 & 0.055 & 0.109 & 0.222 \\
\hline ZSM-12-0.09 & 182.7 & 254.2 & 366.6 & 0.041 & 0.022 & 0.098 & 0.161 \\
\hline
\end{tabular}



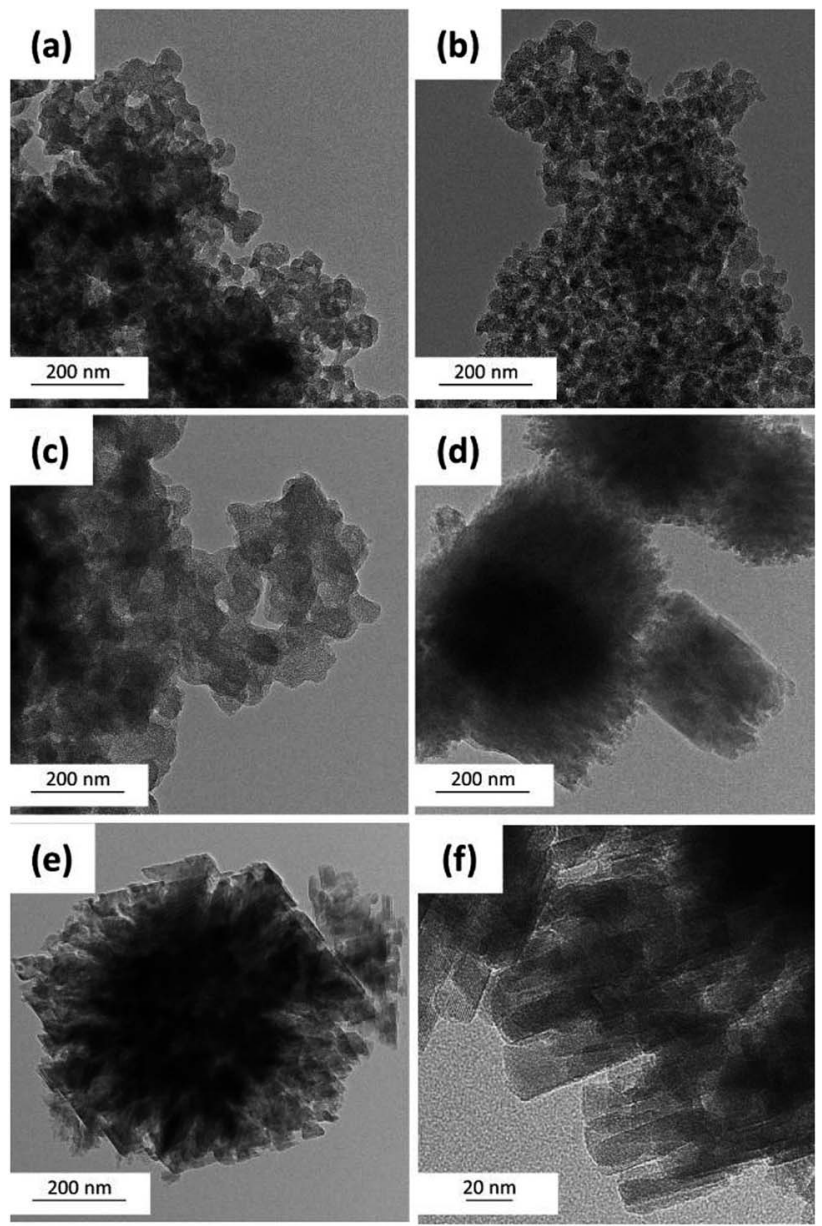

Fig. 6 TEM images of ZSM-12-0.09 samples obtained at different crystallization time: (a) $2 \mathrm{~h}$, (b) $4 \mathrm{~h}$, (c) $6 \mathrm{~h}$, (d) $12 \mathrm{~h}$, and (e and f) $24 \mathrm{~h}$.
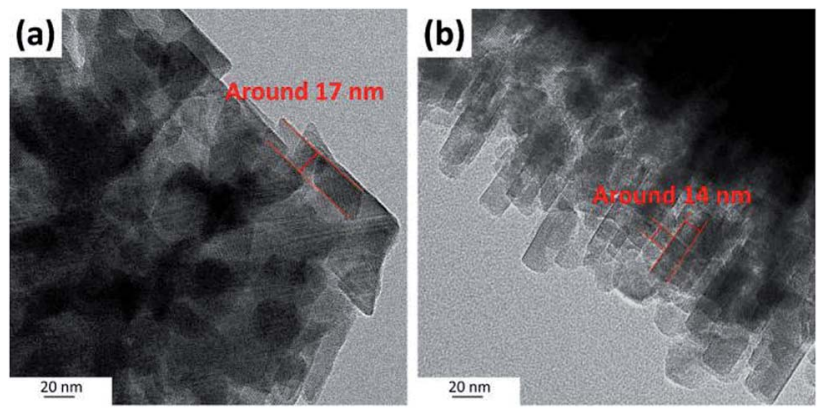

Fig. 7 High-resolution TEM images of ZSM-12 nanolayer sample (ZSM-12-0.09) obtained at crystallization time of $24 \mathrm{~h}$.

In addition, as illustrated in Fig. 7, high-resolution TEM images also confirm that ZSM-12-0.09 obtained at crystallization of $24 \mathrm{~h}$ consists of a multilayer stacking of ZSM-12 nanolayers with a layer thickness around $13-25 \mathrm{~nm}$, which is in accordance with SEM image (Fig. 2(c)).

The degree of networking of $\mathrm{Si}$ atoms in the synthesized samples as a function of crystallization time can be reflected by ${ }^{29}$ Si MAS NMR spectra. As demonstrated in Fig. S2, $\dagger$ a broad peak located in the range between -75 and $-120 \mathrm{ppm}$ was observed for all samples. The broad peak can be divided into three peaks centered at $-111,-99$, and $-92 \mathrm{ppm}$, corresponding to $\mathrm{Q}_{4}, \mathrm{Q}_{3}$, and $\mathrm{Q}_{2}$ species, respectively, where $n$ in $\mathrm{Q}_{n}$ refers to the number of neighbouring $\mathrm{Si}$ atoms that are connected with an oxygen bridge. ${ }^{41,42}$ For instance, the $\mathrm{Q}_{4}$-type $\mathrm{Si}$ atoms are related to $\mathrm{Si}(\mathrm{OSi})_{4}{ }^{43,44}$ The $\mathrm{Q}_{3}$-type $\mathrm{Si}$ atoms can be referred to $\mathrm{Si}(\mathrm{OAl})(\mathrm{OSi})_{3}$ or terminal silanol groups in $\mathrm{Si}(\mathrm{OH})(\mathrm{OSi})_{3},{ }^{41,44}$ whereas the $\mathrm{Q}_{2}$-type $\mathrm{Si}$ atoms are referred to $\mathrm{Si}(\mathrm{OAl})_{2}(\mathrm{OSi})_{2}$ and geminal silanol groups in $\left.\mathrm{Si}(\mathrm{OH})_{2}(\mathrm{OSi})_{2}\right)^{44-46}$ From the results, it is clearly observed that at the initial stage of crystallization $(2 \mathrm{~h})$, the $\mathrm{Q}_{4}$ peak $(-111 \mathrm{ppm})$ is very broad and present as a shoulder peak of the $\mathrm{Q}_{3}$ peak (-99 ppm), suggesting that the coordination environment of the corresponding framework Si atoms is distorted. ${ }^{47}$ As confirmed by SEM image (Fig. 5(a)) and XRD pattern (Fig. 8), the crystalline structure is not formed at this early stage. However, as the crystallization time is increased to 12 and $24 \mathrm{~h}$, the $\mathrm{Q}_{4}$ peak becomes more distinct and narrower, indicating that the silicate network is gradually formed and the degree of $\mathrm{Si}-\mathrm{O}-\mathrm{Si}$ networking becomes higher. Finally, the most distinct Q4 peak is observed for ZSM-12 sample obtained at the crystallization time of $24 \mathrm{~h}$, confirming that the degree of $\mathrm{Si}-\mathrm{O}-\mathrm{Si}$ networking of this sample is the highest compared with other samples obtained at a shorter crystallization time.

As stated above, organosilane surfactants have been extensively utilized to control the structure and the morphology of hierarchical materials because of their amphiphilic properties, allowing us to fine-tune their interaction with zeolitic precursors. ${ }^{40,48,49}$ In order to minimize its surface free energy, the organosilane surfactant creates new surfaces by their selfassemblies, acting as a crystal face inhibitor, which can obstruct the crystal growth in specific direction..$^{50}$ Since TPOAC is composed of hydrolysable methoxysilyl groups $\left(\mathrm{Si}-\mathrm{OCH}_{3}\right)$, aluminosilicate species can react with the hydrolyzed part of TPOAC (Si-OH) during the zeolite synthesis. The interaction between the surfactant molecules and the zeolite precursors can

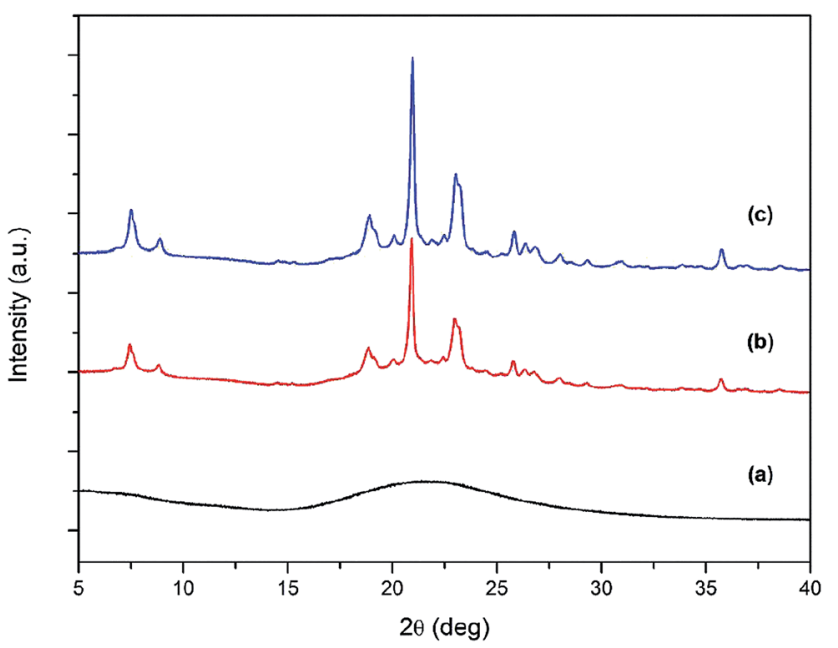

Fig. 8 XRD patterns of ZSM-12-0.09 samples obtained at different crystallization time: (a) $6 \mathrm{~h}$, (b) $12 \mathrm{~h}$, and (c) $24 \mathrm{~h}$. 
initiate the anisotropic crystal growth, eventually leading to the formation of the nanolayers of ZSM-12. ${ }^{31,40}$ During the zeolite crystallization, ZSM-12 nanolayer assemblies are gradually formed. Finally, as the crystallization is prolonged to $24 \mathrm{~h}$, these ZSM-12 nanolayer assemblies become more uniform in company with a higher crystallinity.

\section{Catalytic activity testing}

To demonstrate the advantageous effect of hierarchical ZSM-12 nanolayers, we also performed additional experiments according to the catalytic study of an esterification of levulinic acid (LA) with ethanol to ethyl levulinate (EL). The catalytic performance of synthesized hierarchical ZSM-12 zeolites for esterification of LA to EL was tested in terms of LA conversion (\%) and EL selectivity (\%) achieved after $24 \mathrm{~h}$ of reaction time as shown in Table 3. For hierarchical ZSM-12 nanolayers synthesized in the absence of TPOAC (ZSM-12-0), LA conversion of $50.6 \%$ with EL selectivity of $96.8 \%$ was obtained. This catalyst exhibits activity comparable to the other catalysts reported in the previous literatures. ${ }^{\mathbf{1 , 8 , 5 1}}$ Moreover, the catalytic activity of hierarchical ZSM-12 nanolayer obtained with a TPOAC/SiO $\mathrm{S}_{2}$ ratio of 0.09 (ZSM-12-0.09) is significantly enhanced, as LA conversion and EL selectivity are increased to 78.5 and $98.7 \%$, respectively. It is therefore reasonable to assume that the improved catalytic performance of ZSM-12-0.09 sample is attributed to its nanolayered structure. The formation of such structure can generate interstitial mesopores between layers, enlarging the external surface areas, and thus promoting the molecular accessibility to active sites of zeolite.

As evidenced in Table 1, the external pore volume (due to generated mesopores) is increased from 0.43 to $0.71 \mathrm{~cm}^{3} \mathrm{~g}^{-1}$ when $\mathrm{TPOAC} / \mathrm{SiO}_{2}$ ratio in the synthesis gel is increased from 0 to 0.09. Moreover, pore size distribution (PSD) of ZSM-12-0.09 sample also reveals the additional macropores with a pore diameter around $80 \mathrm{~nm}$ (Fig. S3†). These larger pores are created from the interparticle voids of ZSM-12 nanolayer assemblies and could contribute to the exceptional performance of hierarchical ZSM-12 nanolayer for the bulky-molecule esterification.

The effect of the mesopore volume on the enhanced catalytic activity of the LA esterification can also be confirmed by a plot of LA conversion as a function of the mesopore volume of ZSM-12 (Fig. S4 $\dagger$ ). The results demonstrate that the effect of the mesopore volume on LA conversion is more pronounce at larger

Table 3 Levulinic acid conversion and ethyl levulinate selectivity obtained on different catalysts after $24 \mathrm{~h}$ of reaction (reaction conditions: $T=100^{\circ} \mathrm{C}$, LA : ethanol molar ratio of $1: 1$, catalyst loading of $0.3 \mathrm{~g}$ )

\begin{tabular}{llll}
\hline Sample & Time $(\mathrm{h})$ & $\begin{array}{l}\text { LA conversion } \\
(\%)\end{array}$ & $\begin{array}{l}\text { EL selectivity } \\
(\%)\end{array}$ \\
\hline ZSM-12-0 & 24 & 50.6 & 96.8 \\
ZSM-12-0.09 & 24 & 78.5 & 98.7 \\
ZSM-5 nanosheet & 24 & 52.6 & 98.7 \\
FAU nanosheet & 24 & 58.0 & 95.4
\end{tabular}

mesopore volume. At a lower range of mesopore volume (i.e., smaller than $0.4 \mathrm{~cm}^{3} \mathrm{~g}^{-1}$ ), when the mesopore volume increases for two times (from 0.23 to $0.43 \mathrm{~cm}^{3} \mathrm{~g}^{-1}$ ), LA conversion is improved for only $4.7 \%$ (from $46.1 \%$ to $50.8 \%$ ). On the other hand, at a higher range of mesopore volume (i.e., larger than 0.4 $\mathrm{cm}^{3} \mathrm{~g}^{-1}$ ), when the mesopore volume increases from 0.43 to $0.71 \mathrm{~cm}^{3} \mathrm{~g}^{-1}$, LA conversion is significantly enhanced from $50.8 \%$ to $78.5 \%$. In agreement with the previous works, ${ }^{2,8,20,52}$ a larger pore volume as well as a bigger pore size can decrease the mass transfer resistance, as a larger space is provided for bulky molecules to diffuse inside the zeolite network during the reaction. However, in order to greatly improve the catalytic activity in the LA esterification, the mesopore volume should be large enough to contribute to the mass transfer improvement inside the zeolite structure. Therefore, the mesopores generated from the interparticle voids of ZSM-12 nanolayer assemblies could contribute to the exceptional performance of hierarchical ZSM-12 nanolayer for the esterification involving a large molecule in the reaction pathway.

To proof the concept, an esterification of small carboxylic acid molecule with ethanol was also carried out under the similar experimental condition. It clearly shows that the nanolayered structure has less impact on the catalytic performances in acetic acid esterification, as acetic acid conversion over both ZSM-12-0 and ZSM-12-0.09 are comparable (Table S1†). This result can be explained by the molecular size of the reactants with respect to the pore size of the zeolite. As reported in the literature ${ }^{53}$ the kinetic diameter of acetic acid is $4.4 \AA$, which is smaller than the micropore of ZSM-12 (5.7 $\AA$ × $6.1 \AA)$. Therefore, these small molecules can access to the active sites in zeolite and a larger pore is not required for the mass transfer inside the zeolite pores. On the other hand, the kinetic diameter of levulinic acid is $5.7 \AA^{.54}$ Therefore, these observations confirm that the catalytic performance of a bulky-molecule esterification, which also produces a large molecule of a product, can be greatly improved by the nanolayered structures, which can greatly enhance the accessibility to Brønsted acid sites inside microporous structures.

Furthermore, the catalytic performance for esterification of LA was also evaluated on various zeolite nanosheets with different frameworks. It is worth noting that the synthesis procedures of both ZSM-5 and FAU nanosheets tested herein and their corresponding textural properties were reported in our previous works. ${ }^{34,55}$ For ZSM-5 and FAU nanosheets, they were successfully fabricated by using a single SDA of tetrabutylphosphonium hydroxide (TBPOH) and TPOAC, respectively. The functions of these SDAs are to control the microporous structure of zeolite and generate two-dimensional nanolayers. In contrast, ZSM-12 nanosheet was synthesized by using dualtemplates of CDM and TPOAC. By using CDM as a single template, hierarchical ZSM-12 zeolite could be obtained with nanocrystalline morphology and intercrystalline mesoporosity due to the high affinity of CDM structure with silicate species. However, the introduction of TPOAC as the co-SDA, the morphology of ZSM-12 was transformed from nanocrystals to nanosheet assemblies, and subsequently intercrystalline mesoporosity is improved. Hence, TPOAC is essential for the 
fabrication of hierarchical ZSM-12 nanosheet with enhanced mesoporous feature.

As shown in Table 3, all catalysts show EL selectivity higher than $95 \%$, confirming that EL is the main product of this reaction. Interestingly, hierarchical ZSM-12 nanolayer (ZSM-120.09) exhibits the highest LA conversion, corresponding to $78.5 \%$. The highest catalytic activity of hierarchical ZSM-12 nanolayer could be attributed to the benefit of its microporous structure, composing of a large one-dimensional 12membered ring channel system ${ }^{15}$ and the improved mesoporous feature due to the presence of the secondary SDA.

Although both ZSM-12 and ZSM-5 compose of hierarchical structures and have the similar acid properties (Table S2 $\dagger$ ), the reason of a higher catalytic activity of hierarchical ZSM-12 nanolayer may relate to the benefit of a one-dimensional 12membered ring network, which is also larger than that of ZSM-5 microporous channels. It is well-known that ZSM- 5 consists of two types of interconnecting 10-membered ring channels (straight and sinusoidal channels) with pore openings of $\sim 5.5$ $\AA$. Regarding to the molecular dimension of related molecules with respect to microporous structures of all zeolites, it is suggested that the molecular size of levulinic acid (5.7 $⿱$ ) is close to the pore openings of ZSM-12 $(5.7 \AA \times 6.1 \AA)$ as shown in Scheme 2 . This indicates that the catalytic reaction of levulinic acid esterification with ethanol to ethyl levulinate requires high catalytic spaces. Indeed, FAU exhibits a high acid density, which may have a benefit for an esterification activity (Table S2 $\dagger$ ). However, although its structure is constructed from a large 12membered ring channel system with pore opening of 7.4 $\AA$ and cavity of $13 \AA,^{56}$ it often suffers from disadvantages of its supercage structure, which may inhibit a catalytic activity due to ease of further reactions in a cage-like space, resulting in generating the pore-blocking phenomenon. ${ }^{57}$ This behavior leads to a low catalytic activity even though it contains a high portion of hierarchical porosity (Table S3 $\dagger$ ).

It was stated that the activity of zeolites can be associated with their pore structure and the formation of the transition states of the LA esterification inside the channels. ${ }^{1}$ The mechanism for the acid-catalyzed esterification of carboxylic acids

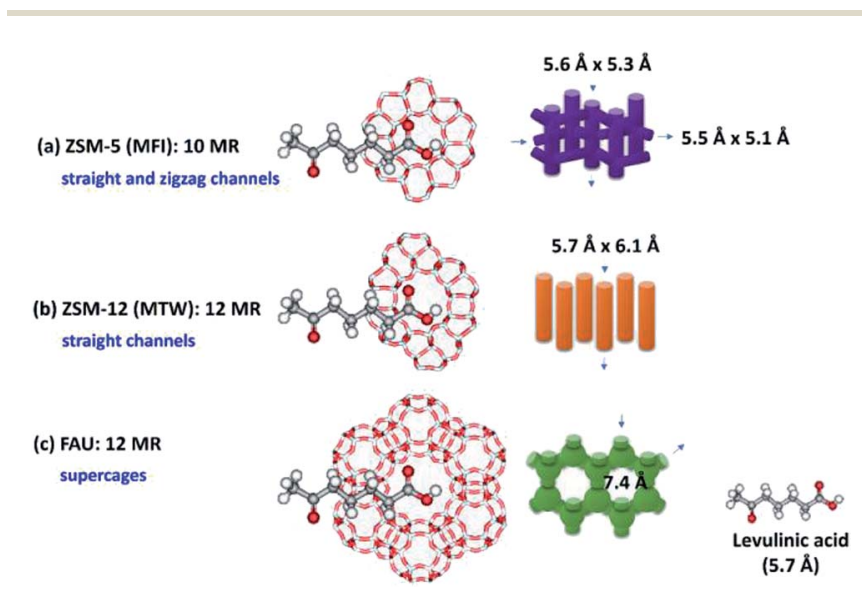

Scheme 2 The molecular size of levulinic acid compared to pore channels of different zeolite frameworks. with alcohols involves the protonation of carboxylic acid and it can be assisted by the Brønsted acid zeolite. With coordination of carboxyl group with the acidic site, the substituent groups could interact with the framework structure. ${ }^{1}$ Hence, the formation of such a transition state might be obstructed by steric hindrance. However, the steric repulsion is considerably less severe, and protonation of the carbonyl oxygen atom is more favorable for zeolite a large pore system without forming large cavities, such as MTW zeolite.

\section{Conclusions}

The nanolayer-assembled ZSM-12 zeolite was successfully prepared by using an organosilane surfactant (TPOAC) as a secondary structure-directing agent. It is demonstrated that the formation of nanolayer structure depends on both TPOAC content and crystallization time. By varying the molar ratio of TPOAC $/ \mathrm{SiO}_{2}$ in the synthesis gel, the TPOAC/SiO $\mathrm{S}_{2}$ molar ratio of 0.09 is the most suitable condition under our synthesis circumstance, as it provides hierarchical ZSM-12 nanolayer assemblies with well-defined structure. In addition, uniform ZSM-12 nanolayer assemblies were observed under the crystallization time of $24 \mathrm{~h}$. Therefore, with the TPOAC/SiO 2 molar ratio of 0.09 and the crystallization time of $24 \mathrm{~h}$, nanolayerassembled ZSM-12 with outstanding properties involving a high fraction of mesopore volumes $\left(V_{\text {ext }} / V_{\text {total }}=0.95\right)$ was obtained.

Interestingly, our designed hierarchical ZSM-12 nanolayers can considerably enhance the catalytic activity for levulinic acid esterification with ethanol in terms of levulinic acid conversion and ethyl levulinate selectivity. The improved activity of hierarchical ZSM-12 nanolayer is related to its enhanced mesoporosity and the presence of a large one-dimensional 12membered ring network, which can promote the accessibility of moleculars to active sites of zeolite. The approach reported herein demonstrates an efficient way to synthesize hierarchical zeolite nanolayer assemblies with ZSM-12 structure via a dual template method and also opens up the interesting aspects for the design of hierarchical zeolites as the heterogeneous catalyst for bulky-molecule reactions like in the case of levulinic acid esterification with ethanol. Moreover, this also extend the design of zeolite nanosheets on other types of zeolites, which has been previously limited to MFI, FAU, and FER types.

\section{Conflicts of interest}

There are no conflicts to declare.

\section{Acknowledgements}

This work was financially supported by the Vidyasirimedhi Institute of Science and Technology, Frontier Research Center (FRC), TTSF research project supported by Thailand Toray Science Foundation, Thailand Research Fund (MRG6180099), and the Office of Higher Education Commission. In addition, this work has been partially supported by the National Nanotechnology Center (NANOTEC), NSTDA, Ministry of Science and 
Technology, Thailand, through its program of Research Network NANOTEC.

\section{References}

1 D. R. Fernandes, A. S. Rocha, E. F. Mai, C. J. A. Mota and V. Teixeira da Silva, Appl. Catal., A, 2012, 425-426, 199-204.

2 C. R. Patil, P. S. Niphadkar, V. V. Bokade and P. N. Joshi, Catal. Commun., 2014, 43, 188-191.

3 Y. Liu, E. Lotero and J. G. Goodwin, J. Catal., 2006, 242, 278286.

4 J. Lilja, D. Y. Murzin, T. Salmi, J. Aumo, P. Mäki-Arvela and M. Sundell, J. Mol. Catal. A: Chem., 2002, 182-183, 555-563.

5 H. E. Hoydonckx, D. E. De Vos, S. A. Chavan and P. A. Jacobs, Top. Catal., 2004, 27, 83-96.

6 J. L. Ropero-Vega, A. Aldana-Pérez, R. Gómez and M. E. NiñoGómez, Appl. Catal., A, 2010, 379, 24-29.

7 A. Sarkar, S. K. Ghosh and P. Pramanik, J. Mol. Catal. A: Chem., 2010, 327, 73-79.

8 Y. Kuwahara, W. Kaburagi, K. Nemoto and T. Fujitani, Appl. Catal., A, 2014, 476, 186-196.

9 J.-Y. Park, Z.-M. Wang, D.-K. Kim and J.-S. Lee, Renewable Energy, 2010, 35, 614-618.

10 J. A. Melero, G. Morales, J. Iglesias, M. Paniagua, B. Hernández and S. Penedo, Appl. Catal., A, 2013, 466, 116-122.

11 Y. Chen, X. Zhang, M. Dong, Y. Wu, G. Zheng, J. Huang, X. Guan and X. Zheng, J. Taiwan Inst. Chem. Eng., 2016, 61, 147-155.

12 F. D. Pileidis, M. Tabassum, S. Coutts and M.-M. Titirici, Chin. J. Catal., 2014, 35, 929-936.

13 A. Corma, Chem. Rev., 1997, 97, 2373-2420.

14 L.-H. Chen, X.-Y. Li, J. C. Rooke, Y.-H. Zhang, X.-Y. Yang, Y. Tang, F.-S. Xiao and B.-L. Su, J. Mater. Chem., 2012, 22, 17381-17403.

15 X. Wei and P. G. Smirniotis, Microporous Mesoporous Mater., 2006, 89, 170-178.

16 J. A. Martens, J. Perez-Pariente, E. Sastre, A. Corma and P. A. Jacobs, Appl. Catal., 1988, 45, 85-101.

17 S. Mehla, K. R. Krishnamurthy, B. Viswanathan, M. John, Y. Niwate, K. Kumar, S. M. Pai and B. L. Newalkar, J. Porous Mater., 2013, 20, 1023-1029.

18 W. Zhang and P. G. Smirniotis, J. Catal., 1999, 182, 400-416. 19 J. Pérez-Ramírez, C. H. Christensen, K. Egeblad, C. H. Christensen and J. C. Groen, Chem. Soc. Rev., 2008, 37, 2530-2542.

20 J. C. Groen, W. Zhu, S. Brouwer, S. J. Huynink, F. Kapteijn, J. A. Moulijn and J. Pérez-Ramírez, J. Am. Chem. Soc., 2007, 129, 355-360.

21 D. Verboekend and J. Pérez-Ramírez, Catal. Sci. Technol., 2011, 1, 879-890.

22 J. Zhou, Z. Hua, J. Shi, Q. He, L. Guo and M. Ruan, Chem.Eur. J., 2009, 15, 12949-12954.

23 J. Zheng, X. Zhang, Y. Zhang, J. Ma and R. Li, Microporous Mesoporous Mater., 2009, 122, 264-269.

24 I. I. Ivanova, I. A. Kasyanov, A. A. Maerle and V. I. Zaikovskii, Microporous Mesoporous Mater., 2014, 189, 163-172.
25 F.-S. Xiao, L. Wang, C. Yin, K. Lin, Y. Di, J. Li, R. Xu, D. S. Su, R. Schlögl, T. Yokoi and T. Tatsumi, Angew. Chem., 2006, 118, 3162-3165.

26 M. B. Yue, M. N. Sun, F. Xie and D. D. Ren, Microporous Mesoporous Mater., 2014, 183, 177-184.

27 K. Na, M. Choi and R. Ryoo, J. Mater. Chem., 2009, 19, 67136719.

28 M. Choi, K. Na, J. Kim, Y. Sakamoto, O. Terasaki and R. Ryoo, Nature, 2009, 461, 246-249.

29 K. Na, M. Choi, W. Park, Y. Sakamoto, O. Terasaki and R. Ryoo, J. Am. Chem. Soc., 2010, 132, 4169-4177.

30 H. Y. Luo, L. Bui, W. R. Gunther, E. Min and Y. RománLeshkov, ACS Catal., 2012, 2, 2695-2699.

31 A. Inayat, I. Knoke, E. Spiecker and W. Schwieger, Angew. Chem., Int. Ed., 2012, 51, 1962-1965.

32 J. Kim, C. Jo, S. Lee and R. Ryoo, J. Mater. Chem. A, 2014, 2, 11905-11912.

33 P. Wuamprakhon, C. Wattanakit, C. Warakulwit, T. Yutthalekha, W. Wannapakdee, S. Ittisanronnachai and J. Limtrakul, Microporous Mesoporous Mater., 2016, 219, 1-9.

34 T. Yutthalekha, C. Wattanakit, C. Warakulwit, W. Wannapakdee, K. Rodponthukwaji, T. Witoon and J. Limtrakul, J. Cleaner Prod., 2017, 142, 1244-1251.

35 S. Salakhum, T. Yutthalekha, M. Chareonpanich, J. Limtrakul and C. Wattanakit, Microporous Mesoporous Mater., 2018, 258, 141-150.

36 K. T. G. Carvalho and E. A. Urquieta-Gonzalez, Catal. Today, 2015, 243, 92-102.

37 K. S. Yoo, S. Gopal and P. G. Smirniotis, Ind. Eng. Chem. Res., 2005, 44, 4562-4568.

38 H. Li, H. Wu and J.-l. Shi, J. Alloys Compd., 2013, 556, 71-78.

39 P. Schneider, Appl. Catal., A, 1995, 129, 157-165.

40 C. Wang, M. Yang, P. Tian, S. Xu, Y. Yang, D. Wang, Y. Yuan and Z. Liu, J. Mater. Chem. A, 2015, 3, 5608-5616.

41 W. Hu, Q. Luo, Y. Su, L. Chen, Y. Yue, C. Ye and F. Deng, Microporous Mesoporous Mater., 2006, 92, 22-30.

42 J. Dedecek, V. Balgová, V. Pashkova, P. Klein and B. Wichterlová, Chem. Mater., 2012, 24, 3231-3239.

43 K. Liu, S. Xie, H. Wei, X. Li, S. Liu and L. Xu, Appl. Catal., A, 2013, 468, 288-295.

44 T. Ikeda, S. Inagaki, T.-a. Hanaoka and Y. Kubota, J. Phys. Chem. C, 2010, 114, 19641-19648.

45 R. Bertermann, N. Kröger and R. Tacke, Anal. Bioanal. Chem., 2003, 375, 630-634.

46 O. Muraza, M. A. Sanhoob and M. A. B. Siddiqui, Adv. Powder Technol., 2016, 27, 372-378.

47 Z. Yan, D. Ma, J. Zhuang, X. Liu, X. Liu, X. Han, X. Bao, F. Chang, L. Xu and Z. Liu, J. Mol. Catal. A: Chem., 2003, 194, 153-167.

48 M. Choi, H. S. Cho, R. Srivastava, C. Venkatesan, D.-H. Choi and R. Ryoo, Nat. Mater., 2006, 5, 718-723.

49 Y. Cheneviere, F. Chieux, V. Caps and A. Tuel, J. Catal., 2010, 269, 161-168.

50 Q. Xiao, Q. Yao, J. Zhuang, G. Liu, Y. Zhong and W. Zhu, J. Colloid Interface Sci., 2013, 394, 604-610.

51 M. Chia and J. A. Dumesic, Chem. Commun., 2011, 47, 1223312235. 
52 X.-R. Chen, Y.-H. Ju and C.-Y. Mou, J. Phys. Chem. C, 2007, 111, 18731-18737.

55 W. Wannapakdee, C. Wattanakit, V. Paluka, T. Yutthalekha and J. Limtrakul, RSC Adv., 2016, 6, 2875-2881.

53 T. C. Bowen, S. Li, R. D. Noble and J. L. Falconer, J. Membr. 56 K. Li, J. Valla and J. Garcia-Martinez, ChemCatChem, 2014, 6, Sci., 2003, 225, 165-176. 46-66.

54 A. Sultana and T. Fujitani, Catal. Commun., 2017, 88, 26-29. 57 J. W. Park and G. Seo, Appl. Catal., A, $2009,356,180-188$. 